\title{
Conhecimento, atitude e prática sobre dengue, seu vetor e ações de controle em uma comunidade urbana do Nordeste
}

\author{
Knowledge, attitude and practice on dengue, the vector and control \\ in an urban community of the N ortheast Region, Brazil
}

Solange Laurentino dos Santos ${ }^{1}$

Ana Catarina dos Santos Pereira Cabral ${ }^{2}$ Lia Giraldo da Silva Augusto ${ }^{3}$

${ }^{1}$ Departamento de M edicina Social, Centro de Ciências daSaúde, Universidade Federal dePernambuco. Av. Prof. M oraes Rego $s / n$, Cidade Universitária. 50670-901 Recife PE. solange.lsantos@ufpe.br

2 Universidade Federal

Rural dePernambuco.

${ }^{3}$ Centro de Pesquisas

Aggeu M agalhães.
Abstract This article presents the results of the study on knowledge, attitude and practice (KAP) on dengue in an urban community in N ortheast of Brazil, and the risk situations involved in its transmission. The study was observational and used a semi-structured survey composed of questions about the disease and vector control measures, which was answered by residents of selected households (IC 95\%) of the community of Santa Rosa, in the city of Cabo deSanto A gostinho, Pernambuco State. The variables of the KAP were classified into appropriate, regular and insufficient. Thereisadequateknowledge about thecharacteristics of the vector and regular regarding the disease and the activities of control. The population does not know that the insecticide used in water is chemical and uses this water for domestic consumption and drinking. Regarding government activity the knowledge was insufficient. It also demonstrates a non appropriate attitude regarding the prevention of dengue and insufficient practice in the prevention of the vector in the household. The practice of water care was adequate for $41 \%$ of residents. The local risk situations raised are related to the intermittency in water and also behavioral.

Key words Dengue, Knowledge, Attitude and practice, Risk situations
Resumo Este artigo apresenta os resultados do estudo sobre conhecimento, atitude e prática (CAP) sobre a dengue, em uma comunidade urbana do N ordeste, e as situações de risco envolvidas com a sua transmissão. 0 estudo foi observacional e utilizou um questionário semi-estruturado, composto por questões sobrea doença, o vetor eas medidas de controle, efoi respondido pel os residentes nos domićlios selecionados (IC 95\%) da comunidadedeSanta Rosa, município do Cabo de Santo Agostinho (PE). As variáveis do CAP foram dimensionadas em adequada, regular einsuficiente. Há um conhecimento adequado sobre as características do vetor e regular no que se re fereà doença eàs atividades de controle. A população desconhece que o larvicida utilizado nos reservatórios deágua éo químico e, ainda, utiliza essa água no consumo doméstico e para beber. Em relação à atividade do governo, o conhecimento dessa população mostrou-seinsuficiente. Demonstra, também, atitude não adequada quanto à pre venção da dengue e prática insuficiente de prevenção do vetor no domicílio. A prática de cuidado com a água mostrou-se adequada para $41 \%$ dos residentes. As situações de risco locais levantadas são relacionadas à intermitência no abastecimento de água e também comportamentais. Palavras-chave Dengue, Conhecimento, Atitude e prática, Situações de risco 


\section{Introdução}

A dengueéuma doença endêmica no Brasil etem sido preocupação das autoridades sanitárias de saúde em todos os municípios brasileiros. Desde 1997, por força do Plano Diretor de Erradicação do Aedes aegypti ${ }^{1}$, está atribuída ao município a responsabilidade pela operacionalização das ações de controle vetorial.

Atualmente, nesse nível de atuação, as práticas de controle do vetor de denguesão realizadas segundo diretrizes do Programa Nacional de Controle da Dengue (PNCD) ${ }^{2}$, cujas estratégias executadas em todas as regiões do país não incorporam a diversidade de características sociais, econômicas, culturais eambientais existentes e tampouco o conhecimento e a percepção da população sobre o problema ${ }^{3}$. Há uma tradição dos modelos de controle que consideram o entendimento da população sobre a doença como homogêneo e direcionam campanhas periódicas padronizadas, como, por exemplo, o "dia D contra a dengue". Estudo recente observou que essa estratégia não éatrativa para a população, sendo o seu desenvolvimento concentrado em áreas específicas e que não atingem a totalidade dos locaisonde severificam necessidades, além demensagens educativas descontextual izadas e ineficazes para impactar a doença e promover ação transformadora ${ }^{4}$.

A idéia hegemônica no M inistério da Saúdeé a de que o controle da dengue só pode ser efetivado pela eliminação do vetor, considerado 0 único elo vulnerável da cadeia de transmissão $0^{1,2}$. Entretanto, as recorrentes epidemias, mesmo na vigência dos programas centrados nas práticas de controle vetorial, têm demonstrado a baixa efetividade dessa estratégia 5 .

0 caráter complexo dessa endemia, devido às inter-relações entre os diversos elementos do ambiente, do homem, do vírus e do vetor, tem sido discutido por diversos autores ${ }^{6-8}$. E vem de monstrando uma necessidade de se repensarem as abordagens de controle adotadas. Nesse sentido, o enfrentamento integrado dos determinantes contextuaistem sido relegado ao segundo plano, em todas as versões proclamadas pelo $\mathrm{Mi}$ nistério da Saúde.

A baixa efetividade das ações de controle da dengue é justificada pelas autoridades sanitárias como de responsabilidade individual da população e da gestão municipal. Donalisío $0^{7}$ considera, no caso da dengue, que existe uma importante subjetividade na compreensão desse fenômeno no seu aspecto coletivo eque énecessário identi- ficar os mecanismos psíquicos, culturais e simbólicos relacionados com as atitudes dos indivíduos na prevenção da doença, e alerta sobre a dificuldade de se fazer essa verificação.

Outros estudos mostraram existir um elevado grau de conhecimento da população sobre dengue e o seu vetor ${ }^{9,10}$. Lefèvre et al. ${ }^{11}$ demonstraram, mediante pesquisa de representação sobre a dengue, que a população do estudo tinha amplo conhecimento sobre a gravidade da doença. Entretanto, em outros contextos sociais, esse conhecimento tem se apresentado distinto, como no estudo de percepção sobre a dengue no qual se observou desconhecimento das situações de risco para a transmissão da doença e para as práticas do uso de inseticidas químicos na eliminação do vetor na formas larvária e adulta ${ }^{3}$.

Em relação ao planejamento, éútil a realização de um diagnóstico situacional das condições existentes, tanto sociais quanto ambientais eculturais, que podem influenciar a transmissão da dengue. O PNCD, por sua verticalidade e padronização operativa, não possibilita que no âmbito comunitário seja internalizada a complexidade envolvida na determinação da dengue; formaliza, pois, para o município, o papel de reprodutor de práticas prescritas nos manuais oficiais.

Assim, a análise da situação de saúde no âmbito local tem sido útil para refletir sobrea influência nessa questão. As incertezas frente à situação de dengue têm levado à necessidade de se pensar modelos que integrem os diversos condicionantes que compõem a complexa causalidade da doença, valorizando-se aspectos socioambientais desse processo. Esse aspecto remeteao que cita Castellanos ${ }^{12}$ sobre a compreensão de saúde como resultante de conteúdos que compõem a noção de "condições de vida" e chama a atenção para a necessidade de se intervir sobre a "rede de acontecimentos" que condicionam a ocorrência de doenças em um ambiente coletivo, devendo isso se dar a partir do conhecimento dos determinantes ali existentes.

Esse autor, ao abordar a questão dos ciclos reprodutivos nos quais operam fatores que favorecem e ou desfavorecem a situação de saúde dos indivíduos e das populações, o faz com propriedade. As intervenções da saúde pública têm como objetivo afetar positivamente a situação de saúde; assim, se predominam fatores negativos, aqui considerados "situações de risco", a situação de saúde se deteriora.

Entende-se o conceito de risco aqui referido não apenas como a medição da ocorrência deeventos, mas reconstruído dentro da teoria da com- 
plexidade, que considera a natureza simbólica, complexa e singular da relação entre saúde-doença-cuidado e os processos sociais, como apresentam Almeida-Filho e Coutinho ${ }^{13}$. Incorporase, também, o conceito de "risco contingencial" como operador do recém-construído campo de práticas da promoção da saúdee da vigilância em saúde, como proposto por esses autores.

Dessa maneira, o objetivo deste trabalho foi avaliar qual o conhecimento, a atitudeea prática relativos à questão da dengue na comunidade de Santa Rosa e, a partir disso, identificar as situações de risco favorecedoras para a transmissão de dengue existentes nessa comunidade.

\section{M étodo}

0 desenho do estudo foi observacional, descritivo, com apoio de um inquérito de conhecimento, atitude e prática (CAP) aplicado aos residentes da comunidade de Santa Rosa, no município do Cabo de Santo Agostinho, localizado no estado de Pernambuco.

Conforme Kaliyaperumal ${ }^{14}$, o estudo CAP mede 0 conhecimento, atitude e prática de uma comunidade e serve como um diagnóstico educacional dessa comunidade. Esse tipo de estudo objetiva reconhecer o que as pessoas sabem sobre um dado tópico, o que sentem sobre esse tema ou alguma idéia pré-concebida que possuam sobre o tema e, por último, a maneira como as pessoas demonstram seus conhecimentos e atitudes através de suas ações.

$M$ arinho et al. ${ }^{15}$ definem conhecimento, atitude e prática com base em estudos similares da seguinteforma: o conhecimento possuído significa recordar fatos específicos ou a habilidade para aplicar fatos específicos para a resolução deproblemas ou, ainda, emitir conceitos com a compreensão adquirida sobre determinado evento. A atitude é, essencialmente, ter opiniões, sentimentos, predisposições e crenças, relativamente constantes, dirigidos a um objetivo, pessoa ou situação. Relaciona-seao domínio afetivo - dimensão emocional. A prática éa tomada de decisão para executar a ação. É o fazer, relaciona-se ao domínio psicomotor afetivo e cognitivo - dimensão social.

Os níveis CAP são um caminho tradicionalmente utilizado pela saúde pública, sobre o qual há maior familiaridade e domínio técnico que, no caso, foi utilizado para uma primeira aproximação com os atores sociais, no sentido de conhecer sua percepção sobre o problema da dengue em seu contexto de vida real.
A metodologia CAP também pode ser utilizada para o diagnóstico da comunidade, após ações educativas, verificando-se as mudanças incorporadas de compreensão dos níveis de conhecimento, atitudee prática, a fim delevar aum processo mais eficiente de conscientização do tema abordado, uma vez que irá permitir um programa de vigilância adaptado mais adequadamente às necessidades da comunidade.

Quanto ao inquérito domiciliar, esse consiste em uma fonte de informação importante para conhecimento da situação de saúde da população; permite, também, a construção de parâme tros para o planejamento e melhoria dos serviços existentes, a incorporação de novas estraté gias de ações, como também instrumental izar os serviços de saúde para desencadearem ações de caráter de preven ção e promoção da saúde ${ }^{16}$.

Nesteestudo, segundo os três tópicos do CAP, observa-seo conhecimento possuído pela comunidade de Santa Rosa, referente à transmissão de dengue, às medidas de prevenção e controle e à participação da comunidade; a atitude adotada pela comunidade em relação às formas de prevenção de dengue e controle de vetor e a prática realizada no domicílio para prevenir criadouros.

0 estudo foi desenvolvido na comunidade de Santa Rosa, localizada no município do Cabo de Santo Agostinho (PE). Essa comunidade foi escolhida por ter um contexto urbano, ser integralmente atendida por uma Equipe do Programa de Saúde da Família (PSF), por apresentar uma clara homogeneidade das condições socioeconômicas e ter uma delimitação geográfica isolada de outras áreas. É constituída de 813 domicílios.

A amostra dos domicílios foi estimada com base na incidência de $50 \%$ de dengue, com um erro aceitável de $5 \%$ para cada intervalo de confiança (IC) de 95\%, obtendo-seo número de 263 domicílios amostrados. Considerando-se a mé dia de quatro residentes por domićlio para 0 município do Cabo de Santo Agostinho, segundo dados do IBGE ${ }^{17}$, a população do estudo ficou composta por 852 pessoas. A amostra foi calculada com uso do programa Epi Info versão 6.04d.

Foi utilizado um questionário semi-estruturado, aplicado nos domicílios selecionados de forma sistemática e respondido pelos responsáveis com idadesuperior a dezesseis anos. No caso em que o domicílio selecionado se apresentou sem morador, no momento da visita, foi selecionado o domicílio imediatamente posterior em ordem crescente. Os dados foram coletados nos meses de julho e agosto de 2007. 
As variáveis foram agrupadas da seguinte maneira: informações sobre condições de moradia e serviços básicos; conhecimento sobre a doença, o vetor e as atividades de prevenção e controle desenvolvidas, tanto individuais quanto governamentais; atitude de preven ção da doença e de controle do vetor e práticas no domicílio para prevenir criadouros e de cuidado com reservatórios de água.

Diversos estudos que aplicaram o CAP utilizaram diferentes formas de valorar as variáveis segundo as três categorias. Nesse estudo, cada variável foi selecionada com o critério de maior força explicativa da seguinte maneira:

- Conhecimento sobre a dengue, quando o morador respondia corretamente sobre as formas de transmissão da doença, as características sazonais, a importância clínica e as ações de prevenção e controle do vetor e de uso de produtos químicos utilizados. Foram estabelecidos os valores de adequado (ou bom), regular e não adequado (ou insuficiente);

- Para a atitude, foram estabelecidos os valores de adequada ou satisfatória e não adequada ou insatisfatória, de acordo com a opinião dada sobreas medidas de controlee prevenção de dengue adotadas e/ou as justificativas dadas;

- Prática na preven ção de criadouros de dengue, em conformidade com as medidas de proteção da saúde e preventiva das situações de risco. Os valores estabelecidos foram: adequada (ou boa), regular e não adequada (ou insuficiente). O Q uadro 1 resume as variáveis, segundo as categorias dimensionadas.

0 estudo permitiu, também, verificar possíveis relações entre a prática de cuidado com a água eo conhecimento. A sel eção da categoria de cuidado com a água se justifica por ser um indicador mais sensível no processo de causalidade da endemia.

A análise estatística incluiu autilização de distribuição de frequência relativa. Para análise comparativa entre as variáveis qualitativas, foi aplicado o teste qui-quadrado ou exato de Fisher (quando necessário). Todas as conclusões foram tomadas ao nível de significância de 5\%. O software utilizado foi SPSS 8.0.

Com base na análise dos dados levantados, foram sendo caracterizadas situações de risco presentes na comunidade do estudo que influenciam, desfavoravelmente, na transmissão de dengue ou na situação de saúde em geral.

0 instrumento utilizado para medir o CAP na comunidade apresentou fragilidades, no que se refere à reduzida capacidade para identificar 0 conhecimento e as atitudes nos níveis mais subjetivos da consciência humana. Entretanto, serviu como uma aproximação da situação existentena comunidade.

Em relação ao viés de informação, é possível que as respostas ao inquérito mostrem uma intenção das pessoas em responder positivamente ao que é esperado para o programa. A limitação ficou no sentido de que com esse instrumento (CAP) não se pode afirmar categoricamente 0 que pensa o sujeito sobre as questões colocadas. Será necessário confrontar as informações obtidas mediante a utilização de outras técnicas, tais como grupo focal e observação participante.

0 estudo foi aprovado pelo Comitê de Ética em Pesquisa do Centro de Pesquisas Aggeu Magalhães, com anuência da Secretaria de Saúde do Cabo de Santo Agostinho e todos os participantes assinaram um termo de consentimento livre e esclarecido, de acordo com a Resolução n 196/ 96 do Consel ho Nacional de Saúde.

\section{Resultados ediscussão}

Características sociais e demográficas, de moradia e serviços básicos

$\mathrm{Na}$ amostra, observou-se que $71 \%$ dos indivíduos têm idade entre 25 e 64 anos, a maioria composta por mulheres (78\%) . 56\% eram pessoas sem participação ativa no mercado de trabaIho, apenas $44 \%$ eram pessoas que têm alguma renda financeira. Em $74 \%$ dos domicílios, residem até quatro pessoas, na sua maioria casal com filhos. As residências têm de cinco a sete cômodos (82\%), caracterizando uma condição aceitável de moradia. O bserva-se a ausência de telas em janelas e portas na maioria das residências.

Cem por cento dos domicílios estão ligados à rede de abastecimento de água, mas essa não é garantida durante todo o dia. Foi referida uma intermitência superior a doze horas. Essa condição leva à prática dearmazenamento deágua para consumo doméstico, o que gera uma "situação de risco" para a transmissão de dengue, por os reservatórios se constituírem em locais privilegiados para a proliferação do mosquito Aedes aegypti. A água desses reservatórios, utilizada para o consumo doméstico, em sua totalidade, é também usada para beber por 91\% (Tabela 1). Em relação ao serviço de col eta delixo, foi referido ser regular para quase a totalidade dos domicílios e há uma prática comum de reutilização de sacos plásticos descartáveis para acondicionamento. 
Quadro 1. Critérios estabelecidos para as categorias de conhecimento, atitude e prática.

1. Informações sobre: condições sociodemograficas, moradia eserviços básicos e D engue

\begin{tabular}{|c|c|c|c|}
\hline \multirow{2}{*}{ 2. Conhecimento } & \multicolumn{3}{|c|}{ Valoração } \\
\hline & Adequado/bom & Regular & $\begin{array}{l}\text { Não adequado/ } \\
\text { insuficiente }\end{array}$ \\
\hline 2.1 Sobre a doença - 5 perguntas & $\begin{array}{l}\text { Quatro a cinco } \\
\text { respostas corretas }\end{array}$ & $\begin{array}{l}\text { Duas a três } \\
\text { respostas corretas }\end{array}$ & $\begin{array}{l}\text { Nenhuma ou uma } \\
\text { resposta correta }\end{array}$ \\
\hline
\end{tabular}

Época de ocorrência

Sintomas

Gravidade

Cuidados e prevenção

\begin{tabular}{|l|c|c|c|}
\hline 2.2. Sobre vetor - 5 perguntas & $\begin{array}{c}\text { Quatro a cinco } \\
\text { respostas corretas }\end{array}$ & $\begin{array}{c}\text { Duas a três } \\
\text { respostas corretas }\end{array}$ & $\begin{array}{c}\text { Nenhuma ou uma } \\
\text { resposta correta }\end{array}$ \\
\hline $\begin{array}{l}\text { Reprodução } \\
\text { Comportamento } \\
\text { Características morfológicas }\end{array}$ & & \\
\hline
\end{tabular}

\begin{tabular}{|l|l|l|l}
\hline 2.3 Atividade de Controle - & Quatro ou mais & Duas a três & Nenhuma ou uma
\end{tabular}
Individual* - 10 perguntas

Tipos de medidas de controle Proteção de reservatórios

Cuidado com ambiente(lixo, água) Tipos produtos de controle Cuidado com o lixo

2.4 Atividade de controle - uso de inseticida - 5 perguntas

\begin{tabular}{|c|c|c|c|}
\hline inseticida - 5 perguntas & respostas corretas & respostas corretas & resposta correta \\
\hline \multicolumn{4}{|l|}{$\begin{array}{l}\text { Finalidade } \\
\text { Efeitos à saúde } \\
\text { Orientações de uso }\end{array}$} \\
\hline $\begin{array}{l}2.5 \text { Atividade Governo* } \\
13 \text { perguntas }\end{array}$ & $\begin{array}{l}\text { Seis ou mais } \\
\text { respostas corretas }\end{array}$ & $\begin{array}{l}\text { Três a cinco } \\
\text { respostas corretas }\end{array}$ & $\begin{array}{l}\text { Nenhuma a duas } \\
\text { respostas corretas }\end{array}$ \\
\hline \multicolumn{4}{|l|}{$\begin{array}{l}\text { Ação realizada } \\
\text { Tipo de ação }\end{array}$} \\
\hline $\begin{array}{l}\text { 2.6 Atividade Agente de Saúde* - } \\
7 \text { perguntas }\end{array}$ & $\begin{array}{l}\text { Quatro ou mais } \\
\text { respostas corretas }\end{array}$ & $\begin{array}{l}\text { Duas a três } \\
\text { respostas corretas }\end{array}$ & $\begin{array}{l}\text { Nenhuma a uma } \\
\text { resposta correta }\end{array}$ \\
\hline \multicolumn{4}{|l|}{$\begin{array}{l}\text { Período de visita } \\
\text { Ação realizada } \\
\text { Tipo ação }\end{array}$} \\
\hline 3. Prática & Adequada/boa & Regular & $\begin{array}{l}\text { Não adequada/ } \\
\text { insuficiente }\end{array}$ \\
\hline $\begin{array}{l}\text { 3.1 Prevenção Vetor no domicílio - } \\
5 \text { perguntas }\end{array}$ & $\begin{array}{l}\text { Quatro a cinco } \\
\text { respostas corretas }\end{array}$ & $\begin{array}{l}\text { Duas a três } \\
\text { respostas corretas }\end{array}$ & $\begin{array}{l}\text { Nenhuma ou uma } \\
\text { resposta correta }\end{array}$ \\
\hline \multicolumn{4}{|l|}{$\begin{array}{l}\text { Identificação vetor } \\
\text { Tipo de Controle }\end{array}$} \\
\hline \multicolumn{4}{|l|}{ 3.2 Cuidado com a água - 5 perguntas } \\
\hline $\begin{array}{l}\text { Proteção reservatórios } \\
\text { Limpeza de reservatórios }\end{array}$ & $\begin{array}{l}\text { Quatro a cinco } \\
\text { respostas corretas }\end{array}$ & $\begin{array}{l}\text { Duas a três } \\
\text { respostas corretas }\end{array}$ & $\begin{array}{l}\text { Nenhuma ou uma } \\
\text { resposta correta }\end{array}$ \\
\hline 4. Atitude & Satisfatória & \multicolumn{2}{|c|}{ Insatisfatória } \\
\hline 4.1 Atitude na prevenção da doença & $\begin{array}{l}\text { opiniões/ } \\
\text { justificativa } \\
\text { adequada }\end{array}$ & \multicolumn{2}{|c|}{ Uma ou nenhuma opinião adequada } \\
\hline $\begin{array}{l}4.2 \text { Atitude no controle do } \\
\text { mosquito da dengue }\end{array}$ & & & \\
\hline
\end{tabular}

*Alguns critérios foram estabelecidos de acordo com a freqüência dos respondentes na variável em questão. 
Tabela 1. Distribuição dos entrevistados quanto as variáveis sociodemográficas, condições de moradia e abastecimento de água na comunidade de Santa Rosa - Cabo de Santo Agostinho, 2007.

\begin{tabular}{|c|c|c|}
\hline Variáveis & $\begin{array}{c}\text { Frequência } \\
\text { N }\end{array}$ & $\%$ \\
\hline \multicolumn{3}{|l|}{ Sexo } \\
\hline Masculino & 56 & 21,7 \\
\hline Feminino & 202 & 78,3 \\
\hline Total & 258 & \\
\hline \multicolumn{3}{|l|}{ Faixa etária } \\
\hline 14 a 24 & 44 & 16,7 \\
\hline 25 a 44 & 108 & 41,1 \\
\hline 45 a 64 & 78 & 29,7 \\
\hline$>64$ & 33 & 12,5 \\
\hline \multicolumn{3}{|l|}{ Ocupação } \\
\hline Desempregado/estudante & 38 & 15,3 \\
\hline Dona de casa & 101 & 40,6 \\
\hline $\begin{array}{l}\text { Atividade remunerada/ } \\
\text { aposentado }\end{array}$ & 110 & 44,2 \\
\hline \multicolumn{3}{|l|}{ Composição da família } \\
\hline casal com filhos & 212 & 81,2 \\
\hline outros tipos de famílias & 20 & 7,7 \\
\hline pessoas solteiras & 29 & 11,1 \\
\hline \multicolumn{3}{|l|}{ Número de cômodos } \\
\hline 1 a 4 & 48 & 18,3 \\
\hline $5 e+$ & 215 & 81,7 \\
\hline \multicolumn{3}{|c|}{ Existe tela em alguma porta/janela } \\
\hline Sim & 5 & 1,9 \\
\hline Não & 255 & 98,1 \\
\hline \multicolumn{3}{|l|}{ Intermitência de água } \\
\hline Até $4 \mathrm{~h}$ & 17 & 6,6 \\
\hline$>12 \mathrm{~h}$ & 100 & 38,9 \\
\hline Em dias alternados & 140 & 54,5 \\
\hline \multicolumn{3}{|c|}{ Armazena água para uso doméstico } \\
\hline Sim & 248 & 99,6 \\
\hline Não & 1 & 0,4 \\
\hline \multicolumn{3}{|l|}{ Armazena água para beber } \\
\hline Sim & 163 & 90,6 \\
\hline Não & 17 & 9,4 \\
\hline
\end{tabular}

Conhecimento sobre a dengue e atividades de controle relativas ao vetor

Todos os entrevistados referiram já ter ouvido falar sobre dengue. A televisão foi citada como a principal fonte de informação. Para $70 \%$, o rádio e o agente de saúde se constituíram outra fonte de informação. A maioria referiu que o domicilio é visitado para orientação sobre dengue, mas apenas 36\% afirmaram realizar alguma atividadede preven ção no seu domicílio. N essesentido, o conhecimento de que a dengue étransmitida pelo mosquito é de domínio da comunidade
(89\%), como também queaépoca demaior ocorrência da doença é na estação chuvosa (78\%) . No entanto, $72 \%$ não souberam apontar os principais sintomas indicativos das situações de agravamento da doença. Sobre os cuidados na pre venção de agravamento, a maioria referiu não conhecer. É possível que o desconhecimento sobre aspectos da doença seja devido à pouca utilização desse conteúdo nas campanhas de divulgação contra a dengue.

Em relação ao conhecimento sobre o vetor $71 \%$ referiram saber dos hábitos diurnos do mosquito, que se reproduz em água limpa, pode transmitir a doença no momento da picada (98\%) e que nem todo tipo de mosquito transmite a doença. Esse conjunto de conhecimentos maior sobre o vetor se deve, provavelmente, ao fato de as ações dos agentes de saúde serem, prioritariamente, centradas no vetor, como também pela maior frequência do tema nas campanhas contra a dengue, o que demonstra uma apropriação pelos moradores dos conteúdos transmitidos.

0 conhecimento sobre as medidas de controleindividual mostrou-se regular para $50 \%$ dos entrevistados, sendo a proteção dos reservatórios de água e a adição de produto na água para eliminar as larvas as medidas mais referidas, enquanto o uso de telas, peixes, mosquiteiros e métodos mecânicos foram pouco citados.

Em relação ao uso de inseticida, não se sabe ser um produto químico, nem édiferenciado das substâncias biológicas. São também desconhecidos potenciais riscos à saúde relacionados a esses produtos. Em decorrência, não se sabe informar os cuidados que devem ser tomados para evitar a exposição química. Esse desconhecimento é possível, devido ao ocultamento de risco nos manuais técnicos, que minimizam esse fato, alegando ser um produto que só faz mal ao mosquito, sendo inócuo ao ser humano ${ }^{18}$. Não obstante, o Programa Municipal deControledeD enguedemonstra preocupação com esses efeitos, uma vez que, há vários anos, autorizou a suspensão da adição em recipientes de água de beber ${ }^{19}$.

Embora a vigilância em saúde ambiental regulamente os padrões de substâncias químicas que representam risco para a saúde, quando colocadas na água de consumo humano $0^{20}$, o pesticida organofosforado Temephos (Abate), que é adicionado na água de consumo doméstico, não consta na lista dessas substâncias. Segundo a Agência de Proteção Ambiental dos Estados Unidos (Environmental Protection Agency - EPA) ${ }^{21}$, o Temephos não deve ser utilizado em água potável e na alimentação. N o entanto, os morado- 
res de Santa Rosa referiram utilizar a água dos reservatórios, que recebeu o inseticida, para consumo doméstico e para beber.

A preocupação com a saúde de populações expostas à água contaminada por substâncias químicas é um tema discutido em fóruns internacionais. 0 relatório da Avaliação Ecossistêmica do Milênio destacou, recentemente, a importância que o consumo de água livre de produtos químicos ede contaminantes microbianos- para cozinhar e se higienizar - tem para a saúde e o bem-estar das pessoas ${ }^{22}$. A opção de controlar 0 vetor da doença com o uso intenso de produtos químicos, ao longo de décadas, é fato preocupante, frente aos potenciais efeitos tóxicos que esses produtos podem ter na saúde humana e nos seres vivos de um modo geral, mediantecontaminação ambiental e alimentar ${ }^{23-26}$. Esses riscos são pouco considerados pela saúde pública, tornando-se um contrassenso para 0 al cance dos objetivos de promoção e proteção da saúde; em contrapartida, promovem o envenenamento sem que se avaliem o impacto sobre a saúde, sua real efetividade e os custos social eambiental que deveriam ser internalizados.

0 conhecimento das atividades realizadas pelo governo em geral não foi adequado; o das atividades dos agentes de saúde, esse se mostrou regular (Tabela 2), o que se percebe pela citação de atividades que não são realizadas pelo programa local, como o uso de peixes e o de outros métodos mecânicos e biológicos.

Atitude de prevenção da dengue e de controle do vetor

A maioria dos entrevistados mostrou atitude insatisfatória quanto à prevenção da dengue em seus diversos aspectos, uma vez que as respostas à pergunta sobre como a dengue pode ser prevenida foram, na sua mai oria, relacionadas ao controledo vetor da doença: "A dengue pode ser prevenida tampando os depósitos com água”; “Limpando a casa e os quintais"; "Colocando areia na planta e cobrindo as caixas d'água”; "Colocando o pozinho na água".

É possível que essa atitude seja um reflexo dos conteúdos das informações veiculadas nos meios de comunicação de massa e dos materiais utilizados nas campanhas de preven ção da dengue. Sabe-se que o foco das campanhas informativas é direcionado para o controle de criadouros do vetor e, no senso comum, prevenir a doença passou a ser entendido como prevenir-se do mosquito. As informações sobre a prevenção e o agravamento da doença, como, por exemplo, a proteção individual e domiciliar contra a picada de insetos, são escassas nos materiais de campanhas utilizados. No aspecto de preven ção primária, o uso de mosquiteiro perdeu, culturalmente, a sua aplicação, além de que, pelos hábitos diurnos do mosquito, não apresenta muita efetividade para insetos adultos; no entanto, pode ser muito útil para prevenção em crianças pequenas, que passam uma grande parte do dia no berço. Diferentemente, no aspecto secundário, a hidratação é uma medida que tem sua efetividade comprovada; entretanto, é pouco adotada e difundida pelos serviços de saúde locais.

Em relação à atitude para o controle do vetor no domicílio, especificamente, a atividade de eliminação da larva, também, se mostrou insatisfatória. A opinião mais referida foi a dejogar aágua fora ou lavar o recipiente. A importância da limpeza específica de criadouros para remover ovos, larvas e pupas de mosquitos é enfatizada em al-

Tabela 2. Distribuição dos entrevistados quanto ao conhecimento sobre a doença, o vetor, atividade de controle individual, de uso de inseticida, do governo, do agente de saúde, prática de cuidado com a água e prevenção do vetor no domicílio.

\begin{tabular}{|c|c|c|c|c|c|c|}
\hline \multirow[b]{2}{*}{ Categorias } & \multicolumn{2}{|c|}{ Adequado/a } & \multicolumn{2}{|c|}{ Regular } & \multicolumn{2}{|c|}{$\begin{array}{l}\text { Não adequado/ } \\
\text { insuficiente }\end{array}$} \\
\hline & $\mathrm{N}$ & $\%$ & $\mathrm{~N}$ & $\%$ & $\mathrm{~N}$ & $\%$ \\
\hline Conhecimento sobre a transmissão da doença & 79 & 30,0 & 172 & 65,4 & 12 & 4,6 \\
\hline Conhecimento sobre o vetor & 172 & 65,4 & 91 & 34,6 & 0 & 0,0 \\
\hline Conhecimento sobre atividade de controle individual & 100 & 38,2 & 132 & 50,2 & 30 & 11,4 \\
\hline Conhecimento sobre uso de inseticida & 86 & 32,7 & 99 & 37,6 & 78 & 29,7 \\
\hline Conhecimento atividade de controle do governo & 68 & 27,8 & 84 & 34,3 & 93 & 38,0 \\
\hline Conhecimento atividade do agente se saúde & 70 & 26,6 & 172 & 65,4 & 21 & 8,0 \\
\hline Prática de cuidado com a água & 105 & 41,3 & 132 & 52,0 & 17 & 6,7 \\
\hline Prevenção vetor no domićlilo & 33 & 12,6 & 103 & 39,5 & 125 & 47,9 \\
\hline
\end{tabular}


guns estudos ${ }^{27,28}$. Sabe-se que antes de eliminar a água com larvas é necessário fazer a correta limpeza dos reservatórios, escovação e colocação de hipoclorito para que todos os ovos que se encontram na superfície possam ser destruídos antes de sejogar a água no ambiente ${ }^{29}$. A penas $4 \%$ citaram comunicar ao agente de saúde a presença de larvas em seu domicílio. A informação da existência de foco na residência éessencial para queo agente de saúde local possa reforçar a orientação naquela residência e nas localidades próximas.

A assimilação da orientação sobre o cuidado com os criadouros com foco de dengue passa pela conscientização dos moradores da importância desse procedimento; esses, no entanto, na maioria das vezes, aguardam que essa ação seja realizada pelos agentes de saúde. Por outro lado, cabe a esses agentes demonstrarem, rotineiramente, a forma adequada de eliminação de larvas e limpeza dos recipientes, considerando que a água, ao ser bem cuidada, evita, além da dengue, outros problemas de saúde.

Foi observado, ainda, que $70 \%$ da população acompanham atividadescomunitárias de prevenção e que apenas $24 \%$ participam de algum grupo formal.

Práticas de prevenção do vetor no domicílio e cuidado com a água

A prática de preven ção do vetor no domicílio foi considerada insuficiente pela maioria dos moradores (48\%). Em relação ao cuidado com a água, essa se mostrou adequada para $41 \%$. 0 conhecimento de que os reservatórios domésticos são os principais criadouros do vetor da denguejáébastantedifundido. Sabe-se, também, que as principais operações de controle vetorial preconizadas pelo PNCD são a inspeção eo cuidado com os reservatórios de água domésticos. Essa operação, feita rotineiramentenos domicílios, deve ser seguida deorientação ecom acompanhamento dos moradores, para que se possa levá-los a uma participação proativa na identificação econtrole de criadouros domésticos. 0 envolvimento comunitário como um elemento de mudança de conduta tem sido avaliado em diversos países e em alguns municípios brasileiros há vários anos $^{30,31}$. Em um estudo realizado para comparar a efetividade de estratégia de mobilização comunitária na redução do índice de infestação, verificou-se maior efetividade no grupo em que havia sido testada a estratégia de mobilização, quando comparado com a área que havia recebido as medidas de controle químico vetorial ${ }^{32}$.
Relação entre prática de cuidado com a água e conhecimento

A prática de cuidado com a água mostrou associação significativa com o conhecimento sobre a doença, $(p<0,003)$, a atividade de controle individual $(p<0,001)$, do governo $(p<0,001)$ edo agente de saúde $(p<0,001)$. A maioria dos que apresentaram prática regular também possuía conhecimento regular sobre a doença $(75,5 \%)$ e sobre a atividade do agente de saúde (87\%). Quanto à prática adequada, essa foi maior para quem possuía conhecimento adequado sobre as atividades de controle individual (63\%) e do governo (60\%). É possível que o fato dese ter bom conhecimento influencie na prática de cuidado com a água. 0 conhecimento sobre o vetor e sobre a atividade de uso de inseticida não mostrou relação com essa prática (Tabela 3). Estudo de $M$ arinho et al. verificou que o desconhecimento das medidas de controle existentes para um problema leva a comportamentos não adequados para a sua prevenção ${ }^{15}$.

Situações de risco identificadas para transmissão de dengue

Há muito tempo, vários estudos têm demonstrado a trama de relações entre os níveis de de terminantes sociais e a situação de saúde de uma população ${ }^{12 ; 33-35}$. Os fatores sociais, econômicos, culturais e comportamentais que influenciam a ocorrência da dengue têm sido utilizados como justificativa para a baixa efetividade de programas de controle implantados e, por isso, considera-se que o levantamento das situações socioambientais existentes no âmbito comunitário deva ser a primeira ação a ser desenvolvida ao se planejar uma intervenção.

Dessa forma, entende-se que as condições de vida e trabalho da população de Santa Rosa influenciam a situação de saúde, em geral, eatransmissão de dengue, em particular. Com base na importância desses condicionantes, como discutido por Castellanos ${ }^{12}$, considera-se a existência de situações de risco para a transmissão de dengue na área de Santa Rosa, tanto no aspecto socioambiental, quanto no comportamental, incorporando o conceito de "risco contingencial" como proposto por Almeida-Filho e Coutinho ${ }^{13}$.

A intermitência de água na comunidade, por mais de doze horas, é a principal condição geradora de possíveis criadouros do vetor da dengue, sendo essa uma condição que não tem me recido a atenção dos gestores locais de saúde. 
Tabela 3. Associação entre prática de cuidado com água e conhecimento sobre a doença, o vetor, atividade de controle individual, de uso de inseticida, do governo, do agente de saúde.

\begin{tabular}{|c|c|c|c|c|c|c|c|}
\hline \multirow[t]{2}{*}{ Categorias } & \multicolumn{2}{|c|}{$\begin{array}{l}\text { Adequado/ } \\
\text { bom }\end{array}$} & \multicolumn{2}{|c|}{ Regular } & \multicolumn{2}{|c|}{$\begin{array}{l}\text { Não adequado/ } \\
\text { insuficiente }\end{array}$} & \multirow[t]{2}{*}{$\begin{array}{l}\text { Valor } \\
\text { de } p\end{array}$} \\
\hline & $\mathrm{N}$ & $\%$ & $\mathrm{~N}$ & $\%$ & $\mathrm{~N}$ & $\%$ & \\
\hline Conhecimento sobre a doença & & & & & & & 0,003 \\
\hline Adequado/bom & 40 & 38,1 & 30 & 22,7 & 5 & 29,4 & \\
\hline Regular & 56 & 53,3 & 100 & 75,8 & 12 & 70,6 & \\
\hline Não adequado/insuficiente & 9 & 8,6 & 2 & 1,5 & 0 & 0,0 & \\
\hline Conhecimento sobre o vetor & & & & & & & 0,805 \\
\hline Adequado/bom & 70 & 66,7 & 85 & 64,4 & 10 & 58,8 & \\
\hline Regular & 35 & 33,3 & 47 & 35,6 & 7 & 41,2 & \\
\hline Não adequado/insuficiente & 0 & 0,0 & 0 & 0,0 & 0 & 0,0 & \\
\hline $\begin{array}{l}\text { Conhecimento sobre atividade de controle } \\
\text { individual do vetor }\end{array}$ & & & & & & & $<0,001$ \\
\hline Adequado/bom & 66 & 62,9 & 29 & 22,0 & 1 & 6,3 & \\
\hline Regular & 32 & 30,5 & 89 & 67,4 & 9 & 56,3 & \\
\hline Não adequado/insuficiente & 7 & 6,7 & 14 & 10,6 & 6 & 37,5 & \\
\hline $\begin{array}{l}\text { Conhecimento sobre atividade de controle } \\
\text { individual de uso de inseticida }\end{array}$ & & & & & & & 0,798 \\
\hline Adequado/bom & 29 & 27,6 & 46 & 34,8 & 5 & 29,4 & \\
\hline Regular & 43 & 41,0 & 47 & 35,6 & 6 & 35,3 & \\
\hline Não adequado/insuficiente & 33 & 31,4 & 39 & 29,5 & 6 & 35,3 & \\
\hline $\begin{array}{l}\text { Conhecimento sobre atividade de controle } \\
\text { do governo }\end{array}$ & & & & & & & $<0,001$ \\
\hline Adequado/bom & 59 & 59,6 & 8 & 6,5 & 0 & 0,0 & \\
\hline Regular & 14 & 14,1 & 64 & 51,6 & 2 & 14,3 & \\
\hline Não adequado/insuficiente & 26 & 26,3 & 52 & 41,9 & 12 & 85,7 & \\
\hline Conhecimento atividade do agente de saúde & & & & & & & $<0,001$ \\
\hline Adequado/bom & 62 & 59,0 & 8 & 6,1 & 0 & 0,0 & \\
\hline Regular & 35 & 33,3 & 115 & 87,1 & 15 & 88,2 & \\
\hline Não adequado/insuficiente & 8 & 7,6 & 9 & 6,8 & 2 & 11,8 & \\
\hline
\end{tabular}

0 desconhecimento dos sintomas de agravamento da dengue é outra condição que pode ser modificada com a inclusão de orientações sobre sinais e sintomas da doença, com linguagem adequada para as pessoas. Martinez-Torres considera importante se conhecer a sequência dos sinais clínicos no diagnóstico dessas diferentes manifestações clínicas. Para ele, éincorreto dizer que a dengue e suas complicações não têm tratamento. $\mathrm{Na}$ verdade, não há um medicamento específico para o agente etiológico, que éum vírus, mas a aplicação de um conjunto de medidas (classificação dos pacientes segundo os sintomas eetapas da enfermidade, reconhecimento precoce dos sinais de alerta da doença) é fundamental para evitar que o paciente chegue a necessitar de unidade de terapia intensiva, uma vez que, nesse momento, às vezes, os tratamentos mais sofisticados e caros podem ser incapazes de salvá- $10^{36}$.
A pouca utilização de métodos de controle mecânico e biológico pelo Programa de Dengue local reflete uma dependência no uso de inseticidas, cujos efeitos à saúdee ao ambiente ainda são pouco conhecidos; apesar de alguns estudos de monstrarem a ação maléfica sobre órgãos e sobre outros seres vivos ${ }^{23-26}$, esses efeitos necessitam ser mais estudados e explicitados para as pessoas. Os moradores ficam à espera de uma ação do agente de saúde para realizar atividades que poderiam ser incorporadas em sua rotina doméstica. $O$ fato de as pessoas da comunidade utilizarem a água com produto químico para beber é outro achado grave, que não tem sido considerado.

È possível que a falta de percepção de risco tanto sobre a doença quanto sobre os produtos químicos influenciena prática insuficientedeprevenção do vetor no domicílio e, também, no cui- 
dado com os reservatórios de água. No caso do cuidado com a água, como medida preventiva de doenças e, em particular, da dengue, apesar de ser um saber antigo, ainda carece de melhor compreensão da comunidade.

A pouca participação em organizações comunitárias, tanto formais quanto informais dos moradores de Santa Rosa é situação de risco que leva à ação pouco proativa dos moradores, frente a questões coletivas que poderiam ser legitimadas com uma maior partici pação e possibilitariam mais poder para a coletividade. 0 baixo exercício de autonomia da comunidade, associado ao comportamento paternalista nas relações com o governo local, impedem o fortalecimento do controle social, uma das diretrizes do Sistema Único deSaúde que tem sido um desafio aosformuladores de políticas públicas no país.

\section{Consideraçõesfinais}

A comunidade deSanta Rosa possui conhecimento adequado sobre o mosquito transmissor da dengue. No entanto, em relação aos sintomas de agravamento da doença eao controle do vetor com medidas deproteção mecânica ebiológica, esseconhecimento se mostrou insuficiente. Demonstra, ainda, atitude não adequada quanto à prevenção da dengue e prática insuficiente de prevenção do vetor no domicílio. As situações de riscos locais levantadas são relacionadas às deficientes condições de saneamento e também comportamentais.

A constatação da ausên cia de conhecimento e de práticas sobre medidas mecânicas de proteção decorre do modelo nacional de controle de dengue, que não incentiva o uso de medidas alternativas para o controle vetorial. Os recursos de compra de insumos são centralizados no $\mathrm{M} \mathrm{i-}$ nistério da Saúde e o gestor municipal não tem autonomia sobre esses recursos, não podendo, assim, usá-los para a adoção de medidas alternativas de controle.

A comunidade de Santa Rosa se mostrou bastante receptiva ao estudo e aberta a novas possibilidades de enfrentamento do problema da dengue. 0 reconhecimento da percepção dos atores locais foi fundamental na aproximação inicial e deveser utilizado no direcionamento deações mais participativas a serem desencadeadas pelos técnicos do Programa Municipal de Dengue. Para isso, é importantequeoutros estudos sejam realizadoseque outros sujeitos sociais que atuam na localidade técnicos do programa, profissionais das unidades de saúde, lideranças comunitárias e tomadores de decisão - também possam se envolver.

O município, com a descentralização das ações de saúde, cada vez mais assume responsabilidades na gestão do SU S. Entre essas, as relacionadas com a promoção e vigilância da saúde. Para tal, além de organizar os serviços de saúde e cuidar do ambiente, são requeridas ações intersetoriais. 0 problema "dengue", portanto, não deve ser uma questão apenas do setor saúde, mas uma questão do governo e da sociedade.

É importante conhecer, em cada contexto sociocultural, como a população reconhece os de terminantes da doença e as formas de prevenção, superar-sea tradição, ainda existente, de campanhas episódicas de informação, em lugar da formação. Nesse sentido, estratégias de educação continuada devem ser desenvolvidas. 


\section{Colaboradores}

SL Santos elaborou o projeto de pesquisa, a coleta de dados, tabulação dos resultados, realizou as análises e escreveu o artigo. ACSP Cabral realizou a categorização do CAP, a tabulação e a análise dos dados. LGS Augusto participou como orientadora da pesquisa, analisou os dados e a revisão crítica do artigo.

\section{Agradecimentos}

À Secretaria de Saúde do Cabo de Santo Agostinho, pela aceitação e colaboração na realização da pesquisa. À Fundação de Amparo à Ciência e Tecnologia do Estado de Pernambuco/Facepe/ M inistério da Saúde/CN Pq, pelo apoio financeiro à pesquisa.

\section{Referências}

1. Brasil. Ministério da Saúde. Fundação Nacional de Saúde. Plano Diretor de Erradicação do Aedes aegypti no Brasil. Brasília: Fundação Nacional de Saúde; 1996.

2. Brasil. M inistério da Saúde. Fundação Nacional de Saúde. Programa Nacional de Controle da Dengue. Brasília: Fundação Nacional de Saúde; 2002.

3. Santos SL. Avaliação das Ações de Controle da Dengue: aspectos críticos e percepção da população - Estudo de caso em um município do Nordeste [dissertação]. Recife (PE): Departamento de Estudos em Saúde Coletiva/NESC/CPqAM/FIOCRUZ; 2003.

4. Sales FMS. Ações de educação em Saúde para prevenção e controle da dengue: um estudo em Icaraí, Caucaia, Ceará. Cien Saude Colet 2008; 13(1):174184.

5. Brasil. Ministério da Saúde. Casos notificados de dengue. Brasil, Grandes regiões e U nidades Federadas. 1997 a 2007. 2008 [site da Internet] [acessado 2008 abr 16]. Disponível em: http://portal.saude.gov. br/portal/arquivos/pdf/tabela_dengue2007.pdf

6. Augusto LGS, Novaes TCP, Abrahão CEC, Pavão CA, Souza AC. Avaliação crítica do Programa de erradicação do Aedes aegypti: contribuições técnicas para medidas de controle. Revista do IM IP 2000; 14(1):90-97.

7. Donalisio MR O Dengue no espaço habitado. São Paulo: Hucitec/FUNCRAF; 1999.

8. Pignatti M G. Saúde e Ambiente: as práticas sanitárias para o controle do dengue no estado de São Paulo. 1985-1995 [dissertação]. Campinas (SP): Departamento de Medicina Preventiva e Social, Universidade de Campinas; 1996.

9. Chiaravalloti N eto F, M oraes MS, Fernandes MA Avaliação dos resultados de atividades de incentivo à participação da comunidade no controle do dengue em um bairro periférico de São José do Rio Preto, São Paulo e da relação entre conhecimento e práticas desta população. Cad Saude Publica 1998; 14(supl 2):101-111.

10. Chiaravalloti Neto F, Fiorin AM, Conversani DT, Cesarino MB, Barbosa AAC, Dibo MR, M orais MS, Baglini V, Ferraz AA, Rosa RS, Battigaglia M, Cardoso Jr. RP. Controle do vetor do dengue e participação da comunidade, São Paulo, Brasil. Cad Saude Publica 2003; 19(6):1739-1749.

11. Lefèvre $A M C$, Ribeiro AF, M arques GRAM , Serpa LLN, Lefèvre $F$. Representações sobre dengue, seu vetor e ações de controle por moradores do Município de São Sebastião, Litoral Norte do Estado de São Paulo, Brasil. Cad Saude Publica 2007; 23(7):16961796.

12. Castellanos PL. Epidemiologia, saúde pública, situação de saúde e condições de vida. Considerações conceituais. In: Barata RB, organizador. Condições de vida e situações de saúde. Rio de Janeiro: Abrasco; 1997. p. 31-75.

13. Almeida-Filho N, Coutinho D. Causalidade, contingência, complexidade: o futuro do conceito de risco. Physis 2007; 17(1):95-137.

14. Kaliyaperumal IEC. Guideline for Conducting a Knowledge, Attitude and Practice (KAP) Study. Commun Ophthalmol 2004; 4(1):7-9. 
15. M arinho LAB, Costa-Gurgel MS, Cecatti JG, Osis MJD. Conhecimento, atitude e prática do auto-exame das mamas em centros de saúde. Rev. Saude Publica 2003; 37(5):576-582.

16. M alta RF, M ishima SM, Almeida M CP, Pereira MJB. A utilização do inquérito domiciliar como instrumento de acompanhamento de ações de saúde em microáreas - analisando a situação vacinal de menores de um ano. Rev. Latino-Am. Enfermagem 2002; 10(1):28-33.

17. Instituto Brasileiro de Geografia e Estatística. Censo demográfico: características da população e dos domicílios. Rio de Janeiro: IBGE; 2000.

18. Brasil. Ministério da Saúde. Fundação Nacional de Saúde. Dengue, instruções para pessoal de Combate ao Vetor. M anual de Normas Técnicas. Brasília: Fundação Nacional de Saúde; 2001.

19. Cardoso MC. Seminário N acional de Controle de Dengue. [comunicação oral]. Recife; 2004.

20. Brasil. M inistério da Saúde. Portaria MS nº 518/2004. Estabelece os procedimentos e responsabilidades relativos ao controle e vigilância da qualidade da água para consumo humano e seu padrão de potabilidade, e dá outras providências. [site da Internet] [acessado 2008 ago 18]. Disponível em: http:// portal.saude.gov.br/portal/arquivos/pdf/portaria_ 518 2004.pdf

21. Environmental Protection Agency. Prevention, Pesticides and Toxic Substances. Temephos facts. [site da Internet] [acessado 2008 ago 28]. Disponível em: http://www.epa.gov/oppsrrd1/REDs/factsheets/ temephosfactsheet.pdf

22. Corvalán C, Hales S, M cM ichael A. Ecosistemas y Bienestar Humano. Síntesis sobre Salud. Un informe de la Evaluación del Milenio (EM). Genebra: Organización Mundial de la Salud; 2005.

23. Repetto R, Baliga SS. Los plaguicidas y el sistema inmunitario: riesgos para la salud publica. Washington, D.C.: WRI; 1996.

24. Sparling DW, Lowe AE, Pinkney AE. Toxicity of Abate to Green Frog Tadpoles. Bull Environ Contam Toxicol. 1997; 58:475-481.

25. Aiub CAF, Coelho ECA, Sodré E, Pinto LFR, Felzenszwalb I. Genotoxic evaluation of the organophosphorous pesticide temephos. Genetics and M olecular Research 2002; 1(2):159-166.

26. Pavão $A C$, Leão $M$. Riscos da carcinogênese química no controle do Aedes aegypti. In: Augusto LGS, Carneiro RM, M artins PH, organizadores. Abordagem ecossistêmica em Saúde: Ensaios para o controle de dengue. Recife: Editora Universitária; 2005. p. 213-225.

27. Perez-Gomez CL, Seda H, Garcia-Rivera EJ, Clark GG. Knowledge and attitudes in Puerto Rico concerning dengue prevention. Rev. Panam. Salud Públ. I Pan Am. J. Public Health 2005; 17(4):243-253.
28. Sherman C, Fernandez EA, Chan AS, Lozano RC, Leontsini E, Winch PJ. La untadita: a procedure for maintaining washbasins and drums free of Aedes aegypti based on modification of existing practices. Am J Trop Med Hyg. 1998; (58)2:257-262.

29. Prefeitura da Cidade do Recife. Guia de Capacitação do Agente de Saúde Ambiental e Controle de Endemias: Curso Introdutório. Recife: Prefeitura da $\mathrm{Ci}$ dade do Recife; 2008.

30. World Health Organization. Evaluation of Communication for Behavioral Impact ("COM BI") Efforts to Control Aedes aegypti Breeding Sites in Six Countries. Geneva: WH O; 2005

31. Perez $D$, Lefrévre $P$, Sánchez $L$, Sánchez $L M$, Boelaert M, Kouri G, Van der Stuyft P. Community participation in Aedes aegypti control: a sociological perspective on five years of research in the health area " 26 de Julio, Havana, Cuba. Trop M ed Int Health 2007; 12(5):664-672.

32. Espinoza-Gomez F, Hernandez-Suárez CM, CollCárdenas R. Educational campaign versus malathion spraying for the control of Aedes aegypti in Colima, Mexico. J epidemiol Community Health 2002; 56(2):148-152.

33. Namikava K, Kikuchi H, Kato S, Takizawa Y, Konta A, lida T, Kimura M. Knowledge, attitudes, and practices of Japanese travelers towards malaria prevention during overseas travel. Travel M ed Infect Dis. 2008; 6(3):137-141.

34. Dahlgren G, Whitehead M. Policies and Strategies to promote social equity in health. Stockholm: Institute for Future Studies; 1991.

35. Buss $P$, Pellegrini Filho A. A saúde e seus determinantes. Physis 2007; 17(1):77-93.

36. Martinez-Torrez E. Atención a enfermos con Dengue. In: Curso Internacional Gestão Integrada de Prevenção e Controle da Dengue. Fortaleza: Secretaria de Vigilância Sanitária; 2004.

Artigo apresentado em 06/10/2008

Aprovado em 19/02/2009

Versão final apresentada em 05/03/2009 